\title{
Improved statistical test for nonstationarity using recurrence time statistics
}

\author{
Christoph Rieke, ${ }^{1,2, *}$ Ralph G. Andrzejak, ${ }^{3}$ Florian Mormann, ${ }^{1}$ and Klaus Lehnertz ${ }^{1}$ \\ ${ }^{1}$ Department of Epileptology, Medical Center, University of Bonn, Sigmund-Freud-Straße 25, 53105 Bonn, Germany \\ ${ }^{2}$ Helmholtz-Institute for Radiation and Nuclear Physics, University of Bonn, Nussallee 14-16, 53115 Bonn, Germany \\ ${ }^{3}$ John-von-Neumann Institute for Computing, Forschungszentrum Jülich, 52425 Jülich, Germany
}

(Received 13 May 2003; published 27 April 2004)

\begin{abstract}
We have recently introduced a measure for nonstationarity using a recurrence time statistic to assess stationarity. In this paper we propose an extension of this method based on a detailed study of the statistics for the case of stationary systems. We derive a simple scheme that allows us to estimate the effective number of degrees of freedom relevant for this statistic. This substantially improves the statistical significance of the method and can be used to improve the significance of various other nonlinear statistics.
\end{abstract}

DOI: 10.1103/PhysRevE.69.046111

PACS number(s): 02.50.-r, 05.45.Tp

\section{INTRODUCTION}

Nonstationarity is a property of a dynamical system that is usually regarded as an unwanted effect in time series analysis. The most common way of dealing with a nonstationary system is to cut the observed time series into short segments during which dynamics of the system can be regarded as approximately stationary. Often essential aspects of the dynamics remain uncovered since cuts are such that characteristic time scales stretch over several segments. Furthermore, evaluation of a characteristic measure may suffer from an insufficient number of data points. The aim of characterizing a dynamical system usually involves analyzing its temporal evolution in state space. The reconstruction of the state space of a stationary system and the identification of related states is a common approach in nonlinear time series analysis and represents a crucial step for these techniques. It has been shown [1] that even for a $D$-dimensional deterministic system that is driven by $P$ slowly time-dependent parameters, a time delay embedding of $m>2(D+P)$ dimensions exists that is sufficient to reconstruct essential aspects of deterministic dynamics.

In an earlier publication [2], we have introduced the loss of recurrence $l^{*}$ to estimate nonstationarity in a nonsegmented time series. The method is based on the analysis of time distances between recurrences. The deviation of the distribution of these distances $\phi_{\text {meas }}$ from a distribution of distances $\phi_{\text {exp }}$ that is expected under stationary conditions allows us to measure nonstationarity. In this context, a system is regarded as stationary if the time index of a neighbor of a point $\vec{x}$ is independent from that of $\vec{x}$. For nonstationarity we expect a deviation: a recurrence is more likely after a short time, when conditions do not yet have changed. We refer to this phenomenon as loss of recurrence. As a measure for the deviation between the observed $\phi_{\text {meas }}$ and the expected distribution $\phi_{\exp }$ we have proposed the median $\mu$ of $\phi_{\text {meas }}$ [2], which is expected to be $\mu_{\text {exp }}=0.5$ for a stationary system. The significance level of the calculated median $\mu_{\text {meas }}$ was specified using the incomplete beta function $I_{\mu}(N / 2, N$

\footnotetext{
*Electronic address: christophrieke@yahoo.com
}

$-N / 2+1)$ with $I_{p}(k, n-k+1) \simeq \sum_{j=k}^{n}\left(\begin{array}{l}n \\ j\end{array}\right) p^{j}(1-p)^{n-j}$. Under the assumption that the measured distribution $\phi_{\text {meas }}$ consists of independent samples $I_{\mu}$ provides the statistical significance of $\mu_{\text {meas }}$ and thus of $l^{*}$. This condition is not completely met, particularly not for oversampled time series, which can lead to spurious detections of nonstationarity. The median of the distribution $\phi_{\text {meas }}$ as a discriminating statistic is a rather robust measure which weakens the effect of dependent samples at the expense of the discriminative power of the test. Furthermore, we have shown the observation time to be a parameter of crucial importance for the analysis of stationarity. Even stationary systems may lead to spurious detections of nonstationarity if the observation time is smaller than the system's characteristic time scales.

Thus, there are two forms of spurious detection of nonstationarity: measurement nonstationarity [3] due to an insufficient observation time and statistical nonstationarity due to an underestimation of statistical fluctuations. The only way to cope with measurement nonstationarity is to increase the observation time. In this paper we pay particular attention to the latter type of nonstationarity, by estimating a confidence interval for our test statistic, i.e., the loss of recurrence, in order to improve its statistical significance.

To deal with oversampled data, reasonable correction schemes have already been proposed [4-6]. These methods, however, are insufficient to correctly determine the statistical significance of a given measure since they do not comprehensively consider the effects concerning correlated or redundant information for a statistical test. One way to estimate a reliable confidence interval is a Monte Carlo simulation using a number of realizations of time series observed from a model system, another way is to measure a large number of time series from the dynamical system to be studied. The first alternative is very time consuming and the second way requires stationarity. Furthermore, it might be impossible to repeat a measurement several times. Note that the estimation of statistical fluctuations on the basis of dependent variables is not only a problem for the loss of recurrence, but is also inherent to other measurements.

This paper is organized as follows. In Sec. II A we propose an extension to our method [2] to correctly estimate the significance of a measured value for nonstationarity that uses 
the Kolmogorov-Smirnov test to discriminate between $\phi_{\text {meas }}$ and $\phi_{\text {exp. }}$. In Sec. II B we discuss the statistical significance of this test and its dependence on the correlation of vectors and nearest neighbors in reconstructed state space. Furthermore, we introduce a correction scheme and investigate its dependence on the embedding parameters, on intrinsic parameters of our measure such as the number of nearest neighbors, and on characteristics of the time series such as the autocorrelation function (Secs. II C and III). The verification for stationary and linear processes and application to stationary and nonstationary model systems is given in Sec. IV, where we demonstrate the generality of the correction scheme by applying it to the nonlinear local prediction error, followed by a conclusion in Sec. V.

\section{METHODS}

\section{A. Loss of recurrence}

The reconstruction of a state space from an observed time series $\left\{x_{i} ; i=1, \ldots, M\right\}$ is usually achieved by the time delay embedding [8] leading to a set of $m$-dimensional vectors $V$ $=\left\{\vec{x}_{n} ; n=1, \ldots, N\right\}$ with $\vec{x}_{n}=\left(x_{n}, x_{n+\tau}, \ldots, x_{n+(m-1) \tau}\right)$, with $m$ and $\tau$ chosen appropriately. For each reference vector $\vec{x}_{r}$ $\in V$, let $\mathcal{U}_{k}\left(\vec{x}_{r}\right)=\left\{\vec{x}_{n}\right\}_{r=1, \ldots, k}$ denote the set of $k$ nearest vectors, with distance defined by the maximum norm $\| \vec{x}_{n}$ $-\vec{x}_{r} \|_{\max }=\max _{i=0, \ldots, m-1}\left|x_{n+i \cdot \tau}-x_{r+i \cdot \tau}\right|$. Let $n_{r}^{i}$ denote the time index of the $i$ th nearest neighbor $\vec{x}_{n_{r}}$ of the reference vector $\vec{x}_{r}$. In the case of stationarity, the time index of a nearest neighbor $n_{r}^{i}$ is a priori independent from that of the reference $r$. Let $l_{r}^{i}=\left|n_{r}^{i}-r\right|$ denote the time lag between $\vec{x}_{r}$ and its $i$ th neighbor. Assuming time translation invariance the expectancy value of the mean time lag is [2]

$$
E\left(l_{r}\right)=\frac{N}{2}-\frac{(r-1)(N-r)}{N-1} .
$$

Following Ref. [4] we exclude neighboring vectors with time lags $l$ less than or equal to the decorrelation time $t$. Let $\phi_{N, r, t}(l)$ denote the a priori expected frequency distribution of the time lag $l_{r}$ of the nearest neighbor under the assumption that for a stationary system each vector (except $\vec{x}_{r}$ itself) has the same probability to be found in the neighborhood of $\vec{x}_{r}$. The distribution function $\Phi_{N, r, t}(l)=\sum_{l^{\prime}=1+t}^{l} \phi_{N, r, t}\left(l^{\prime}\right)$ is the a priori probability that the observed mean time distance is less than or equal to $l$

$$
\Phi_{N, r, t}(l)=\left\{\begin{array}{ccl}
\frac{l-t}{N-1-r-t} & \text { if } t<l \leqslant N-1-r ; \quad r \leqslant t \\
2 \frac{l-t}{N-2 t-1} & \text { if } \quad t<l \leqslant r ; \quad t<r \leqslant \frac{N-1}{2} \\
\frac{l-t+r-t}{N-2 t-1} & \text { if } \quad r<l \leqslant N-1-r ; \quad t<r \leqslant \frac{N-1}{2} \\
2 \frac{l-t}{N-2 t-1} & \text { if } t<l \leqslant N-1-r ; \quad \frac{N-1}{2}<r<N-t-1 \\
\frac{l-t+N-r-1-t}{N-2 t-1} & \text { if } \quad N-1-r<l \leqslant r ; & \frac{N-1}{2}<r<N-t-1 \\
\frac{l-t}{r-t} & \text { if } \quad l \leqslant N-1-r-t ; & r \geqslant N-t-1 .
\end{array}\right.
$$

Since shorter time distances are more likely than longer ones, the frequency functions $\phi_{N, r, t}(l)$ are skewed left sided [cf. Fig. 1(a)], and thus the probability of $l_{r} \leqslant E\left(l_{r}\right)$ is greater than 0.5 , i.e., $\Phi_{N, r, t}\left(E\left(l_{r}\right)\right) \geqslant 0.5$, even for time series with time lags distributed according to $\phi_{N, r, t}(l)$.

In order to solve this problem we map the observed mean time distance to the interval $I=[0,1]$ using the distribution function $\Phi_{N, r, t}(l)$. For the stationary case, the ranks, i.e., the transformed samples $\tilde{l}=\Phi_{N, r, t}(l)$ are uniformly distributed in $I$ and independent of $N$ and $r$, and the probability of $\tilde{l}_{r}$ $\leqslant 0.5$ is equal to 0.5 by construction (cf. Fig. 1). For the nonstationary case the recurrence of state space vectors is reduced for large $l$, since the neighborhood of $\vec{x}_{r}$ depends on the time index $r$, and the indices of the neighboring vectors $\left\{n_{r}^{i}\right\}$ are clustered around $r$. Thus, the observed time distances $l_{r}^{i}$ are on the average smaller than expected. This is also the case for the transformed variables $\tilde{l}$ since $\Phi(l)$ is a strictly monotonic increasing function. The distribution of all transformed time lags $\phi_{\text {meas }}(\widetilde{l})$ reflects the (non)stationarity of the system in the sense that stationarity leads to a uniform distribution, whereas for nonstationarity lower values of $\tilde{l}_{r}$ will accumulate and therefore higher values are reduced. In order to quantify this reduction, we calculated in Ref. [2] the median $\mu_{\text {meas }}$ of $\phi_{\text {meas }}$. The distributions $\phi_{\text {meas }}$ and $\phi_{\text {exp }}$, how- 
a

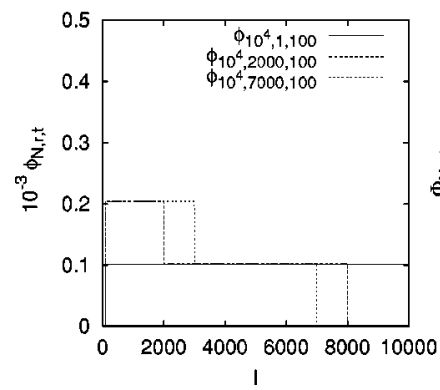

b

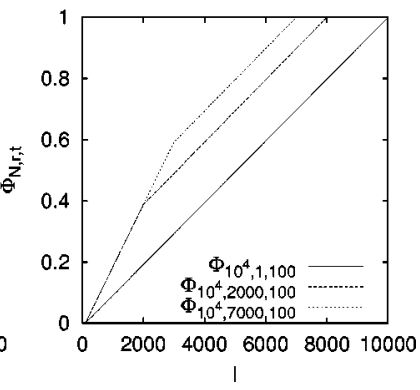

FIG. 1. (a) A priori expected frequency distributions $\phi_{N, r, t}(l)$ and (b) distribution functions $\Phi_{N, r, t}(l)$, respectively, for $N=10^{4}, r$ $\in\{1,2000,7000\}$, and $t=100$ under the assumption of a stationary system.

ever, may differ even though $\mu_{\text {meas }}$ matches the expected median $\mu_{\text {exp }}=0.5$. In this case, the median fails as a discriminating statistic.

In order to improve the sensitivity for a deviation of $\phi_{\text {meas }}$ from $\phi_{\text {exp }}$ we now use the well-known Kolmogorov-Smirnov (KS) test. This statistic is defined as the maximum value of the absolute difference between two cumulative distributions [here, $\Phi_{\text {meas }}(\widetilde{l})$ and $\left.\Phi_{\text {exp }}(\widetilde{l})=\tilde{l}\right]$ :

$$
D_{\text {meas }}=\max _{\tilde{l}}\left|\Phi_{\text {meas }}(\tilde{l})-\Phi_{\exp }(\tilde{l})\right| .
$$

The significance level, i.e., the probability that $D>D_{\text {meas }}$, for $L=N k$ independent samples is approximately given by

$$
P_{L}^{K S}\left(D_{\text {meas }}\right)=Q_{K S}\left(\left[\sqrt{L}+0.12+\frac{0.11}{\sqrt{L}}\right] D_{\text {meas }}\right),
$$

with the function $Q_{K S}(\lambda)=2 \sum_{j=1}^{\infty}(-1)^{j-1} e^{-2 j^{2} \lambda^{2}}$ (cf. Ref. [7], and references therein).

\section{B. Correction schemes}

The requirement of statistical independence of all $L$ samples is not completely met for the set of transformed variables $\left\{\widetilde{l}_{r}^{l}\right\}_{r=1, \ldots, N}^{i=1, \ldots, k}$. In particular, for oversampled data, several correction schemes have been proposed. In Ref. [4] a decorrelation correction for computing the correlation dimension was introduced by requiring a minimum time lag between a reference $\vec{x}_{r}$ and its neighboring vectors $\vec{x}_{n}$. We refer to this scheme as reference-neighbor (RN) correction. This correction scheme was further extended in Ref. [5] to correlated neighboring vectors $\vec{x}_{n}$ and $\vec{x}_{n^{\prime}}$ of the same reference, which we refer to as neighbor-neighbor (NN) correction. A time lag $t$ for the correction schemes is often estimated using the autocorrelation function or the mutual information [9]. The lag $t$ could also be enlarged to $t^{\prime}=t$ $+(m-1) \tau$ to ensure independent vector components leading to an exclusion of neighboring vectors. Another correction scheme was proposed in a false nearest strands method [6] to estimate an optimum embedding dimension. This scheme minimizes redundant information in successive pairs of reference and true neighbors due to temporal correlation in or-

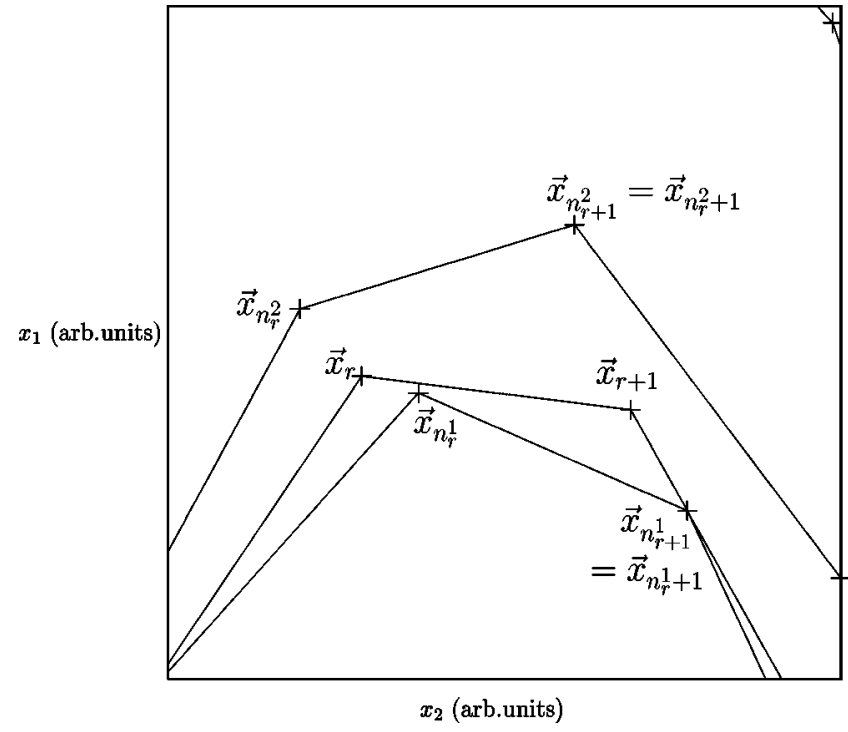

FIG. 2. Relation of neighboring vectors of two successive and correlated reference vectors $\vec{x}_{r}$ and $\vec{x}_{r+1}$ with $\vec{x}=\left(x_{1}, x_{2}\right)$.

der to enhance the proportion of false neighbors. Each strand of successive pairs is identified by its initial pair.

When analyzing the statistical dependencies of a set of reference vectors and their nearest neighbors, we are faced with yet another type of dependencies: In the case of the loss of recurrence, for instance, the set of all $L$ time distances contains redundant information. These occur between recurrence times associated with successive reference points $\vec{x}_{r}$ and $\vec{x}_{r+1}$ (see Fig. 2), but also more generally. Let $L_{r}$ $=\left\{l_{r}^{1}, \ldots, l_{r}^{k}\right\}$ and $L_{s}=\left\{l_{s}^{1}, \ldots, l_{s}^{k}\right\}$ denote the sets of time distances between reference vectors $\vec{x}_{r}$ and $\vec{x}_{s}$ and their particular neighboring vectors $U_{k}\left(\vec{x}_{r}\right)=\left\{\vec{x}_{n_{r}}, \ldots, \vec{x}_{n_{r}}\right\}$ and $U_{k}\left(\vec{x}_{s}\right)$ $=\left\{\vec{x}_{n_{s}}, \ldots, \vec{x}_{n_{s}}\right\}$, respectively. These sets contain redundant information, if $\vec{x}_{r}$ and $\vec{x}_{s}$ are close so that $U_{k}\left(\vec{x}_{r}\right)$ and $U_{k}\left(\vec{x}_{s}\right)$ overlap. To minimize the redundancy we may choose independent reference vectors $r=1, t^{\prime}, 2 t^{\prime}, 3 t^{\prime}, \ldots$ or even halve or quarter the data. This preselection, however, might reduce the number of independent variables too much leading to an insufficient sampling of the systems' dynamics, which may result in unforeseen problems and pitfalls. The correction scheme proposed in Ref. [6], however, does not completely correct redundant information to provide statistical independence of all time lags. In addition, there exists a mutual neighborhood of a reference vector $\vec{x}_{r}$ and its neighboring vectors, e.g., if $\vec{x}_{r} \in U_{k}\left(\vec{x}_{n_{r}^{i}}\right)$, then $l_{r}^{i}$ is equal to $l_{n_{r}^{i}}^{j}$ for one $j$ $\in\{1, \ldots, k\}$. The $k+1$ sets $L_{r}, L_{n_{r}^{1}}, \ldots, L_{n_{r}^{k}}$, each consisting of $k$ time distances obtained from $\vec{x}_{r}$ and each neighboring vector $\vec{x}_{n_{r}} \in U_{k}\left(\vec{x}_{r}\right)$ as reference, will altogether contain at least $k$ independent time distances. In this case, including another neighbor would not provide additional information.

In order to cope with redundant information within all $L$ time distances we here propose a correction of $L$ to approximate the number of the degrees of freedom of the statistical test used in this study, i.e., the KS test. The maximum number of all, not necessarily independent, time distances is $L$ $=k N$. Due to an overestimated number of degrees of free- 
a)

\section{1: without correction}

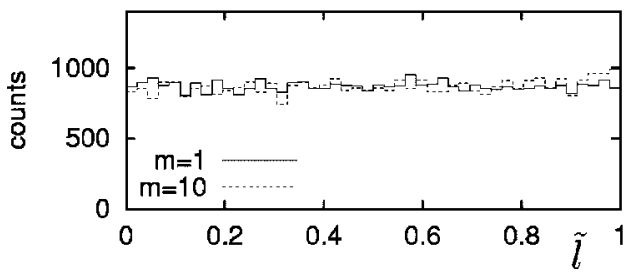

2: with $R N$-correction $t^{\prime}=20$

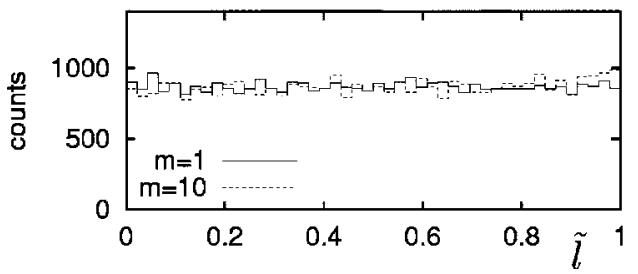

3: with $R N$ - and $N N$-correction $t^{\prime}=20$ b)
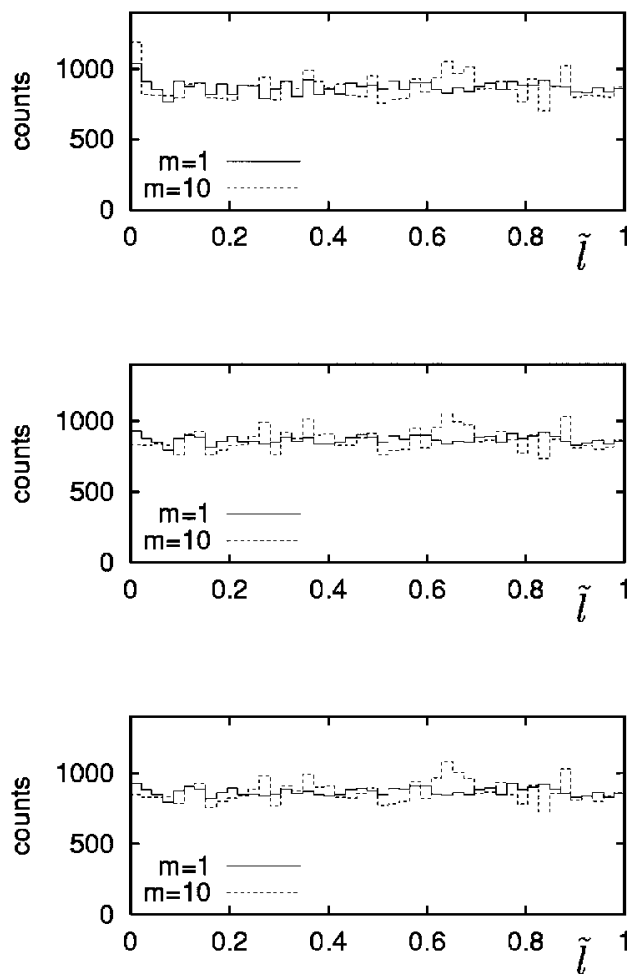

FIG. 3. Histograms of $\tilde{l}$ for time series $M(1)$ (left column) and $M(10)$ (right column) with $k=4$ neighboring vectors for embedding dimensions $m=1$ and $m=10$ and different correction schemes. From top to bottom: (1) without correction, (2) with RN-correction, and (3) with RN and NN corrections.

dom, the empirical size $p$ of the discriminative statistic is larger than the nominal size $\alpha$. As a lower bound for a corrected number of independent variables we propose $L_{\text {min }}$ $=L /(k+1)[t+(m-1) \cdot \tau]$. For the particular case of the $\mathrm{KS}$ test, we therefore replace the number of degrees of freedom $L$ of the discriminative statistic by $L_{\min }: P_{L_{\text {min }}}^{K S}\left(D_{\text {meas }}\right)$. In this way the empirical size is smaller than the nominal size.

\section{Investigation of dependencies of the significance on parameters}

In order to examine the statistics of the proposed test we use simple stationary stochastic time series. These were generated by moving average processes $M(Q)$ defined by $x_{n}$ $=1 / Q \sum_{i=1}^{Q} \eta_{n-i}$ with $Q \geqslant 1$ and $\eta_{n-i}$ denoting independent and identically distributed Gaussian random variables $\left[\eta_{n-i}\right.$ $\in \mathcal{N}(\kappa, \sigma)$ with mean $\kappa=0$ and standard deviation $\sigma=1]$. $M(1)$ is the uncorrelated Gaussian random process. In the following we set the time delay $\tau=1$.

Figure 3 shows the influence of different correction schemes on $\phi_{\text {meas }}(\widetilde{l})$ for time series $M(1)$ and $M(10)$ with $N=10^{4}$ data points, embedding dimensions $m=1$ and $m=10$ and $k=4$ neighboring vectors calculated (1) without any correction scheme, (2) with NR correction, (3) with NR and NN corrections. In Fig. 3(b1) the frequency of short distances is increased due to closeness of subsequent vectors in state space. The variance of the distributions increases with both $m$ and the order $Q$ of the $M(Q)$ process caused by an increased correlation of the variables. RN correction reduces the increased frequency in the first bin of the histogram particularly for $m=10$ whereas the NN correction reduces the variance of the distributions. The effect of the latter is marginal for $k=4$ neighboring vectors, but becomes more pronounced for an increased number of neighbors. In order to quantify the influence of these correction schemes, we estimated the empirical size $p$ using 1000 realizations of the processes $M(1)$ and $M(10)$ (cf. Table I).

Without any correction schemes, the empirical size $p$ exceeds the nominal size $(\alpha=0.05)$ for the KS test $P_{L}^{K S}\left(D_{\text {meas }}\right)$ even for the Gaussian random process $M(1)$ with embedding dimension $m=1$. Furthermore, $p$ tends towards higher values with increasing $m$ and increasing $Q$. Correction schemes reduce the empirical size $p$, which, however, remains well above $\alpha$. After correction of the number of variables to $L_{\text {min }}$, the empirical size $p$ tends to zero $\left(p \leqslant 10^{-3}\right)$ which is markedly lower than $\alpha$. Thus, the number of false rejections is diminished, the discriminative power, however, might be reduced as well. The number $L_{\min }$ is an estimation of a lower bound of the number of degrees of freedom leading to $p$ $\leqslant \alpha$. The number of all variables $L=N k$ on the other hand represents an estimation of the upper bound with an empirical size $p$ greater than or equal to the nominal size $(p \geqslant \alpha)$. The performance of the discriminative statistics is optimal 
TABLE I. Empirical size $p$ using the KS statistics at a nominal size of $\alpha=0.05$ from 1000 realizations for each process $M(1)$ and $M$ (10) with $N=10^{4}$, embedding dimension $m=1$ and $m=10$ and $k$ $=5$ nearest neighbors. $p$ was estimated using different correction schemes, i.e., without $\mathrm{RN}$ correction $\left(t^{\prime}=0\right)$ and with $\mathrm{RN}$ correction $\left(t^{\prime}=20\right)$ as well as without and with $\mathrm{NN}$-correction. The minimum time lag of $t^{\prime}=20$ is greater than or equal to the sum of the embedding dimension and the decorrelation time of the $M$ process.

\begin{tabular}{lccccccc}
\hline \hline \multicolumn{1}{c}{$m$} & \multicolumn{3}{c}{1} & & \multicolumn{3}{c}{10} \\
\hline$R N$ correction & 0 & \multicolumn{2}{c}{20} & 0 & \multicolumn{2}{c}{20} \\
$N N$ correction & 0 & 0 & 20 & 0 & 0 & 20 \\
$M(1)$ & 0.13 & 0.151 & 0.134 & 0.811 & 0.817 & 0.808 \\
$M(10)$ & 0.267 & 0.16 & 0.168 & 0.979 & 0.934 & 0.911 \\
\hline \hline
\end{tabular}

when the theoretical distribution of $P_{L_{e f f}}^{K S}\left(D_{\text {meas }}\right)$ matches the empirical distribution function $\Phi\left(D_{\text {meas }}\right)$, i.e., the empirical size $p$ matches the nominal size $\alpha(p \approx \alpha)$. This requirement defines the effective number of degrees of freedom $L_{e f f}$, which we expect to satisfy $L_{\text {min }} \leqslant L_{e f f} \leqslant L$.

\section{Estimation of the effective number of degrees of freedom $L_{e f f}$}

A Monte Carlo simulation allows us to estimate the effective number of degrees of freedom $L_{e f f}$. In this way we fit the theoretical distribution function $P_{L}^{K S}\left(D_{\text {meas }}\right)$ of the KS test to the empirical distribution function $\Phi_{K S}\left(D_{\text {meas }}\right)$ of the Monte Carlo simulation via the number of degrees of freedom $L$. The distance of both distributions is defined similar to the KS test by

$$
D(L)=\underset{D_{\text {meas }}}{\max }\left|\Phi_{K S}\left(D_{\text {meas }}\right)-P_{L}^{K S}\left(D_{\text {meas }}\right)\right| .
$$

Finally we define an estimate $\hat{L}_{e f f}$ of the effective number of degrees of freedom $L_{e f f}$ by

$$
D\left(\hat{L}_{e f f}\right)=\min _{L}\{D(L)\}
$$

Figure 4 shows $D(L)$ for $M(1)$ and $M(10)$ time series with $N=10^{4}$ and for embedding dimensions $m=1$ and $m=10$. The global minimum $\hat{L}_{e f f}$ of $D(L)$ depends on the autocorrelation function of each process and more obviously on $m$. Estimates of the global minimum are displayed in Table II.

Figure 5 confirms the closeness of the theoretical frequency function $p_{\hat{L}_{\text {eff }}}^{K S}\left(D_{\text {meas }}\right)$ of the KS test with the empirical frequency distribution $\phi^{K S}\left(D_{\text {meas }}\right)$.

In order to provide an approximation $L_{q} \simeq L_{e f f}$, we focus on the dependencies of $L_{e f f}$ on the embedding dimension $m$, the number of neighboring vectors $k$, and the decay of the autocorrelation function $\rho(\nu)$ characterized by $\min _{\nu}\{\nu: \rho(\nu)$ $\left.\leqslant \rho(0) / e^{2}\right\}$ as an appropriate value for the decorrelation time $t$. Defining
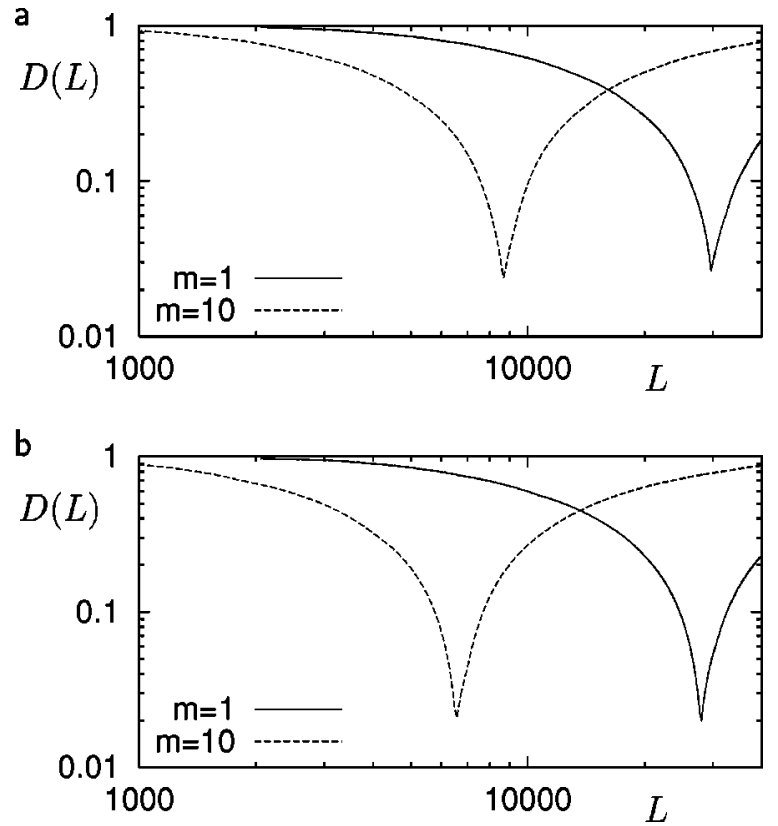

FIG. 4. Distance $D(L)$ between the empirical distribution of the KS test $\Phi_{K S}\left(D_{\text {meas }}\right)$ and the theoretical distribution function in dependence on the number of degrees of freedom $L$. The distribution function $\Phi_{K S}\left(D_{\text {meas }}\right)$ from (a) the $M(1)$ process and (b) the $M(10)$ process were estimated both with 200 realizations using embedding dimensions $m=1$ and $m=10, k=4$ nearest neighbors, and $N=10^{4}$ data points leading to $L=4 \times 10^{4}$.

$$
q_{e f f}=\frac{L}{L_{e f f}},
$$

we expect $q_{e f f}$ to be independent of $N$ since $L_{e f f} \propto N$.

\section{RESULTS}

In order to examine $q_{e f f}(m, k, t)$ we estimate $\hat{q}_{e f f}=L / \hat{L}_{e f f}$ for 200 realizations each of $M(Q)$ processes with $Q$ $\in\{1,2,5,10,20,50,100\}$. Furthermore we varied the embedding dimension $m=\operatorname{int}\left(\sqrt{2}^{n}\right)$ with $n=1, \ldots, 14$ and the number of nearest neighbors $k=1,2,3,4$ at $N=10^{4}$.

Figure 6 shows a selection of the estimated correction factors $\hat{q}_{\text {eff }}$ in dependence on $m$. The results of these simulations reflect the dependencies of the effective number of degrees of freedom $L_{e f f} \propto 1 / q_{\text {eff }}$ on $t, m$, and $k$. The following simple relationship:

$$
q(m, k, t)=0.5 \cdot \sqrt{(1+k)\left(m^{2}+t\right)},
$$

approximates $q_{\text {eff }}$ which is indicated by lines $(k=1,2,4)$ in Fig. 6. Equation (8) is chosen such that $q(m, k, t) \geqslant q_{e f f}$, i.e.,

TABLE II. Number of degrees of freedom $L_{m i n}, \hat{L}_{e f f}$, and $L$.

\begin{tabular}{lcccccc}
\hline \hline & \multicolumn{2}{c}{$L_{\text {min }}$} & \multicolumn{2}{c}{$\hat{L}_{\text {eff }}$} & \multicolumn{2}{c}{$L$} \\
\hline & $M(1)$ & $M(10)$ & $M(1)$ & $M(10)$ & $M(1)$ & $M(10)$ \\
$m=1$ & 8000 & 800 & 29650 & 28030 & 40000 & 40000 \\
$m=10$ & 800 & 421 & 8660 & 6580 & 40000 & 40000 \\
\hline \hline
\end{tabular}




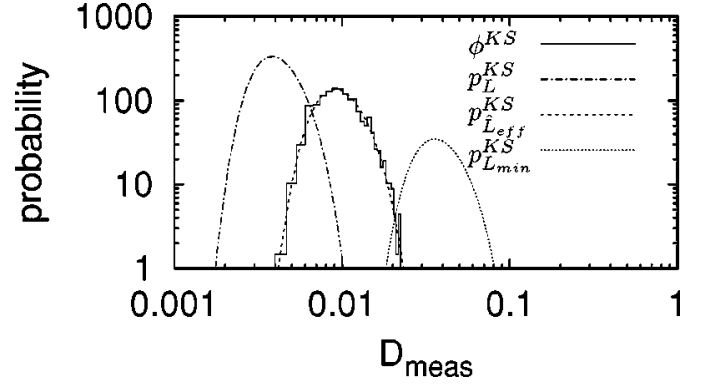

FIG. 5. Empirical frequency distribution $\phi^{K S}\left(D_{\text {meas }}\right)$ of 200 realizations of the $M(10)$ process estimated with $N=10^{4}, m=10$, and $k=4$ and the theoretical frequency functions $p^{K S}(L), p^{K S}\left(\hat{L}_{e f f}\right)$, and $p^{K S}\left(L_{\text {min }}\right)$.

the empirical size is less than or equal to the nominal size $(p \leqslant \alpha)$. Note that even for the $M(1)$ process at an embedding dimension of $m=1$ the estimated divisor $\hat{q}_{\text {eff }}$ is $>1$. This effect is due to a mutual neighborhood of the reference and its neighboring vectors, since each vector in state space is an independent realization of a $\mathcal{N}(0,1)$ process. This approximation defines the approximated effective number of degrees of freedom $L_{q}=L / q(m, k, t)$.

As a verification of our ansatz [Eq. (7)] we also estimated the dependence of $q_{\text {eff }}$ on $N \in 5 \times 10^{3}, \ldots, 10^{5}$, which turned out to be approximatively constant as expected. This remarkable effect leads to the following conclusion: Although the correction scheme proposed [6] is reasonable in a false nearest neighbors method, identifying a strand of successive pairs of references and nearest neighbors by its initial pair does not correct the number of degrees of freedom sufficiently.

The decreasing length of strands with increasing $N$ is in contrast to the fact that the effective number of degrees of freedom is proportional to $N$. Instead, the approximation of the number of degrees of freedom using a divisor $q_{\text {eff }}$ improves the statistical significance for the measure such as the loss of recurrence.

\section{APPLICATION TO STATIONARY AND NONSTATIONARY NONLINEAR MODEL SYSTEMS}

Up to now we have demonstrated the statistics of $D_{\text {meas }}$ on stationary, linear stochastic processes. As a next step we will apply this method to nonlinear, stationary, and nonstationary model systems in order to validate its empirical size and, in particular, to examine its discriminative power.

The first nonlinear deterministic model is a generalization of the baker's map (cf. Ref. [10]):

$$
\begin{gathered}
\text { if } v_{n} \leqslant a: \begin{array}{c}
u_{n+1}=b u_{n} \\
v_{n+1}=v_{n} / a,
\end{array} \\
\text { if } v_{n} \geqslant a: \begin{array}{c}
u_{n+1}=0.5+b u_{n} \\
v_{n+1}=\left(v_{n}-a\right) /(1-a) .
\end{array}
\end{gathered}
$$

In the following we take $a=0.4$. We obtain a stationary system if $b=0.5(B 1)$, and two nonstationary systems by slowly varying $b$ according to $b=0.4+(0.2 / M) n(B 2)$ and
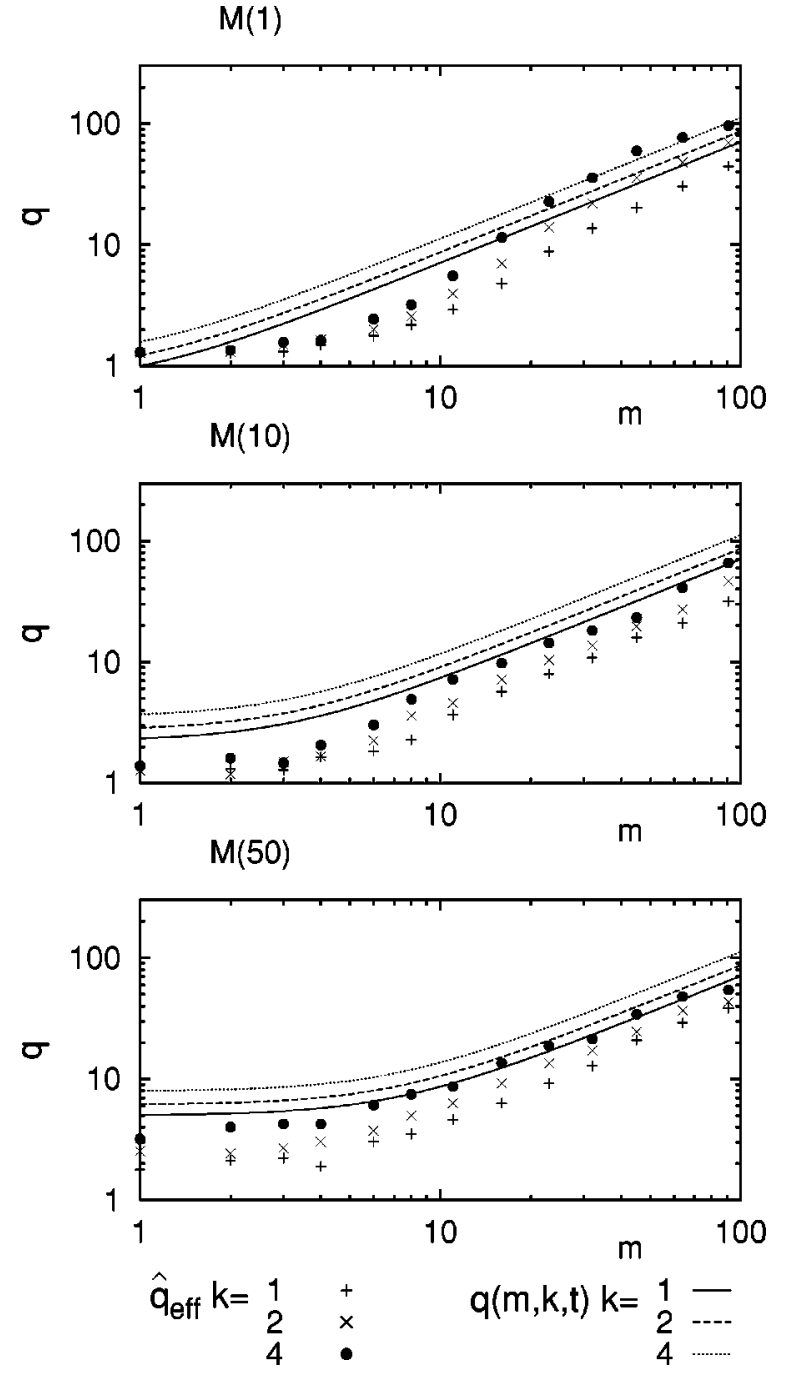

FIG. 6. Estimated divisor $\hat{q}_{\text {eff }}$ (symbols) in dependence on the embedding dimension $m$ for different numbers of nearest neighbors $k \in\{1,2,4\}$ and different processes: (a) $M(1)$, (b) $M(10)$, and (c) $M(50)$. Approximation $q(m, k, t)$ of $q_{\text {eff }}$ using Eq. (8) added as different lines, solid $(k=1)$, dashed $(k=2)$, and dotted $(k=4)$.

$b=0.2+(0.6 / M) n(B 3)$, respectively, with $M=10^{5}$. We record the sum $w_{n}=u_{n}+v_{n}$, subtract the running mean, and normalize to running unit variance within an interval of 20 data points.

As a second nonlinear deterministic model system, we examine the Lorenz system [11]:

$$
\begin{gathered}
\frac{d x}{d t}=a(y-x), \\
\frac{d y}{d t}=r x-y-x z, \\
\frac{d z}{d t}=x y-b z,
\end{gathered}
$$

with $a=10, b=\frac{8}{3}$. For $25 \leqslant r \leqslant 90$ this system exhibits chaotic behavior. We calculate data vectors $(x, y, z)$ for fixed time 

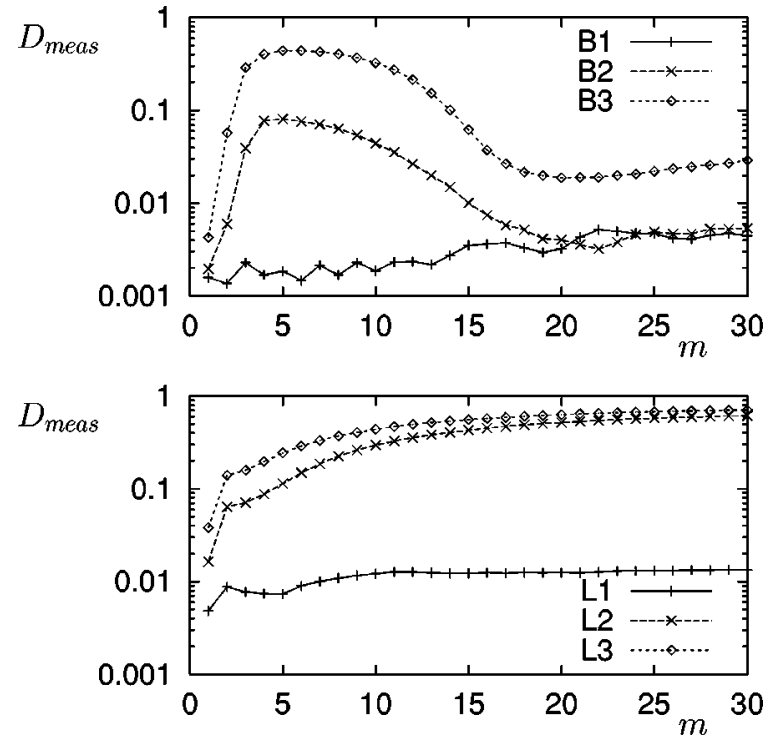

FIG. 7. Distance $D_{\text {meas }}$ in dependence on the embedding dimension $m$ with $k=4$ nearest neighbors and $N=10^{5}$ data points, for one realization of stationary $(B 1)$ and nonstationary $(B 2, B 3)$ baker's map (upper part) and stationary $(L 1)$ and nonstationary $(L 2, L 3)$ Lorenz system (lower part).

intervals of $\Delta t=0.01$. In the following, we will focus on the $x$-component $\left\{x_{n}\right\}$. The stationary time series is generated with $r=25(L 1)$ and the nonstationary time series are generated by slowly varying the parameter $r=25+(25 / M) n(L 2)$ and $r=25+(65 / M) n(L 3)$, respectively, for $M=10^{5}$.

First of all we choose an appropriate embedding dimension for the systems by analyzing the dependence of $D_{\text {meas }}$ on $m$ (cf. Fig. 7). An insufficient embedding dimension leads to false recurrences and thus distorts $\phi_{\text {meas }}(\widetilde{l})$ to a uniform distribution. For stationary systems $D_{\text {meas }}$ is almost independent of $m$. Nonstationary systems exhibit a nonmonotonic dependence of $D_{\text {meas }}$ on $m$. A local maximum of $D_{\text {meas }}$ can be observed at values coinciding with the lower bound of embedding dimensions as suggested in Ref. [1]. For the Lorenz system $D_{\text {meas }}$ increases over a large range of $m$.

As a next parameter we investigate the observation time by varying the number of data points $N$, in order to examine the time scales of the dynamic. Figure 8 shows the empirical frequency distribution $\phi\left(D_{\text {meas }}\right)$ for different stationary processes and systems at different observation times. For time series $M(10)$ and $B 1$, the empirical frequency function matches the respective theoretical frequency function $p^{K S}\left(L_{q}\right)$ with $L_{q}$ degrees of freedom. For the $M(10)$ process, $\phi\left(D_{\text {meas }}\right)$ matches the theoretical function $p_{L_{q}}^{K S}$ at all investigated observation times (cf. Fig. 8). Furthermore, our correction scheme is validated even for the stationary baker's map $(B 1)$. In Fig. 9 empirical distributions $\phi\left(D_{\text {meas }}\right)$ are represented by their mean and standard deviation. The confidence interval $C_{\alpha}(L)$ of a one-sided test with $\alpha=0.1$ and with corrected number of variables $\left[C_{0.1}\left(L_{q}\right)\right.$ (lower lines) and $C_{0.1}\left(L_{\min }\right)$ (upper lines)] are also included. The empirical distribution $\phi\left(D_{\text {meas }}\right)$ of the stationary stochastic processes and the stationary baker's map confirm $C_{0.1}\left(L_{q}\right)$ as a reliable con-
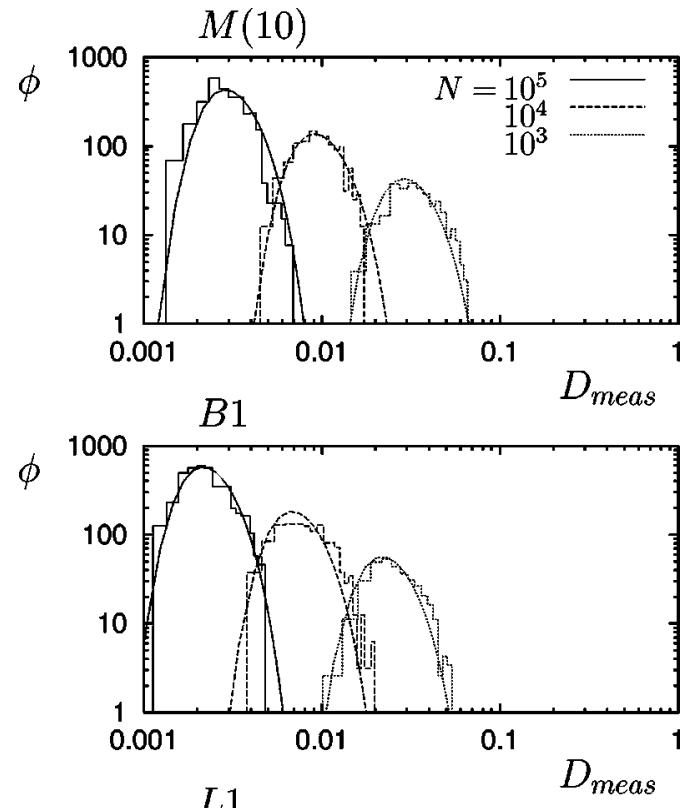

$\phi$

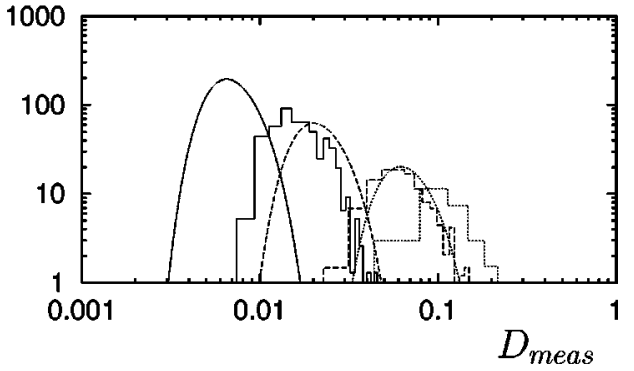

FIG. 8. Empirical frequency distribution $\phi^{K S}\left(D_{\text {meas }}\right)$ of 500 realizations of the $M(10)$ process with $m=10$, the stationary baker's map (B1) with $m=6$, and the stationary Lorenz system ( $L 1)$ with $m=24$ all estimated using $k=4$ nearest neighbors and different observation times $N=10^{3}, N=10^{4}$, and $N=10^{5}$ (steps). The theoretical frequency functions $p^{K S}\left(L_{q}\right)$ are depicted as different lines.

fidence interval. In contrast $L_{\min }$ underestimates the number of degrees of freedom.

Figure 9 shows further that the distributions of $D_{\text {meas }}$ at $N=10^{4}$ for the nonstationary systems overlap with $D_{\text {meas }}$ for the respective stationary system. A discrimination is impossible. With increasing observation time, $D_{\text {meas }}$ increases for the nonstationary systems $(B 2, B 3, L 2, L 3)$ and decreases for the respective stationary system $(B 1, L 1) . B 3$, for instance, is identified as nonstationary for $N \geqslant 2 \times 10^{4}$ using the confidence interval $C_{0.1}\left(L_{q}\right)$.

For the stationary Lorenz system $L 1$, there is a significant deviation between $p_{L_{q}}^{K S}\left(D_{\text {meas }}\right)$ and $\phi\left(D_{\text {meas }}\right)$. Furthermore, $C_{0.1}\left(L_{q}\right)$ is smaller than the mean of $\phi\left(D_{\text {meas }}\right)$ for all $N$. Thus, $L_{q}$ overassesses the number of degrees of freedom, whereas $C_{0.1}\left(L_{\min }\right)$ appears to be more reliable. This could be attributed to an insufficient observation time, although Fig. 9 shows no convergence of $C_{0.1}\left(L_{q}\right)$ with $\mu+2 \sigma$ of $\phi\left(D_{\text {meas }}\right)$. On the other hand, $t$ might be underestimated due to the finite number of data points used for the autocorrelation estimate.

In order to obtain a better estimate of the autocorrelation function $\rho(\nu)$ and thus of $t$ we increased the observation time 


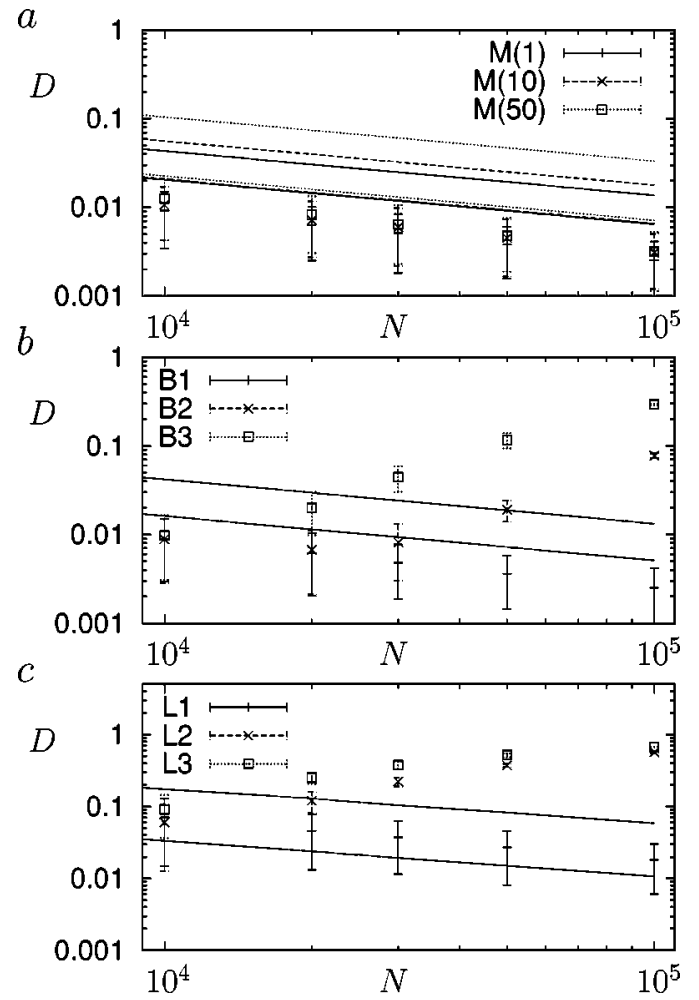

FIG. 9. $D_{\text {meas }}$ in dependence on the observation time $N$ (in data points) for (a) stochastic processes $M(1), M(10)$, and $M(50)$; (b) stationary $(B 1)$ and nonstationary baker's maps ( $B 2$ and $B 3)$, and (c) stationary ( $L 1)$ and nonstationary ( $L 2$ and $L 3)$ Lorenz system. Mean of $\phi\left(D_{\text {meas }}\right)$ as points and $2 \sigma$ in error bars, estimated from 500 realizations with embedding parameters $m=10$ (a), $m=6$ (b), and $m=24$ (c) with $k=4$ neighboring vectors. The confidence interval $C_{0.1}(L)$ of the theoretical distributions $p^{K S}$ for $L_{q}$ and $L_{\min }$ as number of degrees of freedom at a significance level $\alpha=0.1$ for a one-sided test is plotted as lines.

up to $N=10^{7}$ (cf. Fig. 10). The differences between the estimates for different data length $N$ reflect the effect of an insufficient observation time. The first zero crossing of the autocorrelation function strongly depends on $N$. Even at $N$ $=10^{7}, \rho(\nu)$ appears to be estimated incompletely. The approximated effective number of degrees of freedom $L_{q}$ $=L / q(m, k, t)$ is thus overestimated, particularly at small observation times since $t$ depends on $N$. The observed nonstationarity of the Lorenz system $L 1$ is caused by an insufficient observation time and might be classified as measurement

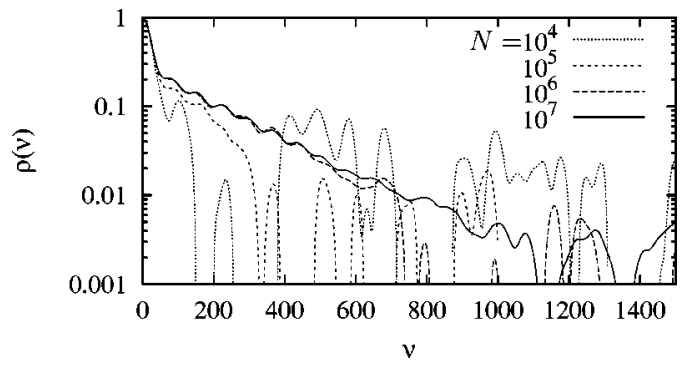

FIG. 10. Autocorrelation function $\rho(\nu)$ of the $x$ component of the Lorenz system using different lengths $N$ of the time series. nonstationarity. Thus, in order to discriminate between stationary and nonstationary systems, both a reliable confidence interval and an adequate observation time are required.

In the following we apply our proposed correction scheme to the local nonlinear mean prediction error $\mathcal{P}$ (cf. Ref. [3]). $\mathcal{P}$ represents the average over many individual errors $\mathcal{P}_{r}$ and thus is likely to be Gaussian distributed. The empirical errors, however, are not expected to be independent, which complicates the estimation of the variance of $\mathcal{P}$ (cf. Ref. [10]). Thus we use our correction scheme to improve its estimation.

The mean prediction error $\mathcal{P}$ is defined as the mean of the set of all individual prediction errors $\mathcal{P}_{r}$ for each reference $\vec{x}_{r}$ :

$$
\mathcal{P}=\frac{1}{N-h} \sum_{r=0}^{N-1-h} \mathcal{P}_{r}
$$

where the individual error is the difference of the future of the reference and the future of its nearest neighbor $\vec{x}_{n_{r}}$ :

$$
\mathcal{P}_{r}=\left|\frac{\vec{x}_{r+h}-\vec{x}_{n_{r}+h}}{m}\right|
$$

$h$ denotes the prediction horizon (here $h=20$ ). The standard deviation $\sigma_{P}$ of the frequency distribution of the individual prediction errors $\mathcal{P}_{r}$ is given by

$$
\sigma_{\mathcal{P}}=\sqrt{\frac{1}{N-h} \sum_{r=0}^{N-1-h} \mathcal{P}_{r}^{2}-\mathcal{P}^{2} .}
$$

According to the central limit theorem, the mean $\mathcal{P}$ of $L$ independent variables is approximately Gaussian distributed with standard deviation $\sigma_{\mathcal{P}} / \sqrt{L}$. We now define $\delta$ as the relative error of the mean

$$
\delta=\frac{\sigma_{\mathcal{P}}}{\mathcal{P} \sqrt{L}}
$$

which is the standard deviation $\sigma_{\mathcal{P}} / \sqrt{L}$ normalized by the mean. The number of all individual errors is $L=(N-h) k$. We use our correction scheme to improve the significance of $\delta$. In order to verify the estimated and corrected $\delta$, we simulate the standard deviation of $\mathcal{P}$ from 200 realizations of the stationary dynamical systems analyzed before $(B 1, L 1)$.

Figure 11 shows a comparison of $\delta$ obtained both from a Monte Carlo simulation and from an estimation using $L_{\text {min }}$, $L_{q}$, and $L$ as different numbers of degrees of freedom. When compared to the Monte Carlo simulation, the relative error of the mean $\delta$ with $L_{q}$ degrees of freedom exhibits a reliable estimate for the system $B 1$. The uncorrected relative error is by far too low. For the system $L 1$, the number of degrees of freedom $L_{q}$ turned out to be overassessed, similar to the results for the loss of recurrence as discussed previously. For the nonstationary systems we expect similar relative errors when compared to the respective stationary system. The absolute mean prediction error $\mathcal{P}$, however, might be increased due to the change of the dynamics. 

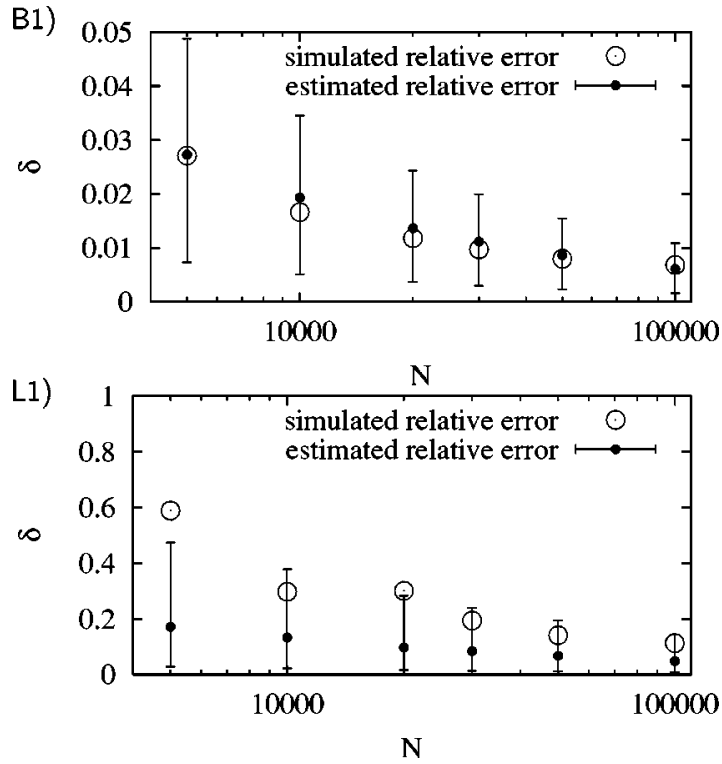

FIG. 11. Relative statistical error of the nonlinear local prediction error in dependence on the number of data points $N$ for systems $(B 1)$ and $(L 1)$. Errors $\delta$ were calculated using 200 realizations of a Monte Carlo simulation for each observation time (empty circles). Estimated error $\delta$ with $L_{q}$ degrees of freedom (solid circles) and with $L_{\text {min }}$, respectively, $L$ as number of degrees of freedom (error bars).

\section{CONCLUSION}

We have discussed in detail the statistical significance of our recently proposed technique for measuring nonstationarity from unpartitioned time series. To assess correctly its significance we have introduced a scheme which allows us to estimate the effective number of degrees of freedom. We have demonstrated the effects on different parameters, i.e., the embedding dimension and the autocorrelation of a time series of the observed system. Furthermore, we have verified the statistical dependence of our correction scheme on the number of data points of the observed time series. A simple recipe allows us to approximate this correction scheme in order to provide a reliable confidence interval. The concept of estimating the effective number of degrees of freedom for a statistic of reference vectors and their neighboring vectors in reconstructed state space is, however, more general, which we exemplified using the local nonlinear prediction error as another measure.

The reconstruction of the state space combined with a nearest neighbor algorithm is a widespread concept in nonlinear time series analysis. Thus a corrected number of variables as number of degrees of freedom improves the confidence interval of the statistical fluctuations for other nonlinear statistics. Finally, our method allows us to determine a suitable observation time, to trace characteristic time scales, and to quantify nonstationarity in observed systems. Further studies are underway which apply our improved statistical test to a variety of time series from different physical models (see, e.g., Ref. [12]) and which aim to extent preliminary findings obtained from experimental data [13]. A crucial dependence on the observation time for detection of nonstationarity of nonlinear model systems still remains, i.e., the observation time should be sufficient to cover all essential time scales of the system.

\section{ACKNOWLEDGMENTS}

We thank Peter David and Peter Grassberger for valuable comments in earlier versions of this manuscript. This work was supported by the Deutsche Forschungsgemeinschaft.
[1] R. Hegger, H. Kantz, L. Matassini, and T. Schreiber, Phys. Rev. Lett. 84, 4092 (2000).

[2] C. Rieke, K. Sternickel, R. G. Andrzejak, C. E. Elger, P. David, and K. Lehnertz, Phys. Rev. Lett. 88, 244102 (2002).

[3] H. Kantz and T. Schreiber, Nonlinear Time Series Analysis (Cambridge University Press, Cambridge, 1997).

[4] J. Theiler, Phys. Rev. A 34, 2427 (1986).

[5] J. Farmer and J. Sidorowich, in Evolution, Learning and Cognition, edited by Y.-C. Lee (World Scientific, Singapore, 1989), pp. 277-330.

[6] M. B. Kennel and H. D.I. Abarbanel, Phys. Rev. E 66, 026209 (2002).

[7] W. H. Press, S. A. Teukolsky, W. T. Vetterling, and B. P. Flannery, Numerical Recipes in $C$ (Cambridge University Press,
Cambridge, 1992).

[8] F. Takens, in Dynamical Systems and Turbulence, edited by D. A. Rand and L.-S. Young (Springer-Verlag, Berlin, 1980), Vol. 898, pp. 366-381.

[9] A. M. Fraser and H. L. Swinney, Phys. Rev. A 33, 1134 (1986).

[10] T. Schreiber, Phys. Rev. Lett. 78, 843 (1997).

[11] E. Lorenz, J. Atmos. Sci. 20, 130 (1963).

[12] A. Witt, J. Kurths, and A. Pikovsky, Phys. Rev. E 58, 1800 (1998).

[13] C. Rieke, R. G. Andrzejak, F. Mormann, T. Kreuz, P. David, C. E. Elger, and K. Lehnertz, IEEE Trans. Biomed. Eng. 50, 634 (2003). 